\title{
VI.
}

\section{Ueber die Anwendung einiger neuerer Mittel in der Obrenheilkunde.}

\author{
Von \\ Dr. F. Kretschmann, \\ Privatdocent in Halle a. 8 .
}

(Von der Redaction ubernommon am 25. Januar 1889.)

Wohl in keinem Zweig der gesammten Medicin findet sich so häufig Gelegenheit, kurzdauernde, aber sehr schmerzhafte Operationen auszuführen, als auf dem Gebiete der Ohrenheilkunde, und sehr berechtigt ist daher der Wunseh, ein Mittel zu besitzen, das eine Anästhesie herbeizufihhren vermag, ohne bei der Application den umständlichen Apparat zu benöthigen, welchen die Chloroformnarkose erfordert: Lagerung des Kranken auf das Operationsbett, sachkundige Assistenz, grossen Zeitaufwand u. s. w. Ein solches Mittel schien mir das Bromäthyl') nach den mehrfachen ginstigen Berichten, welche über dasselbe vorliegen, zu sein. Ich habe es in einigen 20 Fällen angewendet und kann mich den bisherigen anerkennenden Aeusserungen voll und ganz anschliessen.

Ausgefuihrt wurde dis Bromäthylnarkose bei Spaltung von Furunkeln, Extraction von Polypen, galvanokaustischen Kanterisationen, Auslöffelung von cariösen Höhlen, Paracentesen. Die Operationen wurden sämmtlich während der Sprechstunde gemacht unter Assistenz eines nicht besonders sachkundigen Gehülfen. Die Patienten verliessen ausnahmslos nach wenigen Minuten das Zimmer ohne wesentliche Beschwerden.

Das Bromäthyl (Aethylum bromatum) ist eine farblose ätherische Flissigkeit von 1,39 specifischem Gewicht, welche bei

1) Das Bromătbyl ist streng genommen nicht unter die neueren Mittel zu zählen. Vor ungefähr 30 Jahren wurde es mehrfach benutzt, gerieth aber bald in Vergessenheit und wurde erst in den letzten Jabren aufs Neue angewandt und besonders fur kurzdauernde Operationen empfohlen. 
$39^{\circ}$ C. siedet. Mit Wasser geschüttelt reagirt es neutral. 1) Durch Licht erfährt es eine Zersetzung, welche nicht durch Veränderung seiner Farbe sich kenntlich macht, sondern dadurch, dass es mit Wasser geschüttelt saure Reaction zeigt. Bei einem derartig zersetzten Präparate nimmt der Kork nach einigen Wochen eine bräunliche Färbung an, bedingt dureh die Einwirkung des freiwerdenden Broms. In dunklem Glase aufbewahrt und vor Lichtstrahlen geschützt, hält sich das Präparat Monate lang unverändert und wirksam. Der Kork blcibt weiss und wird in keiner Weise arrodirt. Einige Tropfen Bromäthyl auf die Hand gegossen verdampfen in wenigen Secunden, ohne einen Rückstand oder ein fettiges Gefubl zu binterlassen. Durch die schnelle Verdunstung wird ähnlich, wie bei Aether, ein erhebliches Kältegefühl hervorgerufen. Die Inhalationsmaske bedeckt sich nach einigen Minuten Gebrauches mit kleinen weissen nadelfòrmigen Krystallen. Die Dämpfe des Bromäthyl sind nicht so leicht entzündlich, wie diejenigen des Aether, und es kann deshalb das Präparat obne Scheu bei Lampenlicht angewendet werden.

Der Geruch des Bromäthyl ist dem des Chloroform nicht ganz unähnlich, erinnert aber auch gleichzeitig an den Geruch von säuerlichen Aepfeln. Ein scharfer oder stechender Geruch des frischen Präparates lässt auf Verunreinigung schliessen. Erst nach längerer Zeit macht sich auf der Maske, welche zur Narkotisirung benutzt wurde, ein scharfer knoblauchähnlicher Geruch bemerkbar, der sich auch im Zimmer, in welehem die Narkose vorgenommen wurde, findet.

Es ist wichtig, alle diese chemischen Eigenschaften des Bromäthyls zu kennen, da man nur auf diese Weise sicher sein kann, ein reines Präparat zu haben, und ein absolut reines Präparat ist durchaus nothwendig, wenn man sich nicht Misserfolgen aussetzen will.

Abgesehen ron gelegentlichen absichtlichen oder unabsichtlichen Verunreinigungen oder Verfälschungen von Seiten der Producenten ist zu warnen vor Verwechselungen des Bromäthyls mit dem Bromäthylen, die infolge der ähnlich klingenden Namen leicht eintreten können. Szumann (a. a. O.) macht darauf besonders aufmerksam und giebt die Eigenschaften des Bromäthylen an als einer farblosen, angenehm riechenden Flïssigkeit von 2,163

1) Szumann, Das Bromäthyl und die Bromäthylnarkose. Therapeut. Monatshefte. 1888. S. $155 \mathrm{ff}$. 
spec. Gewicht und $129^{\circ} \mathrm{C}$. Siedepunkt. Eine Verwechselung mit diesem Präparat ist um so verhängnissvoller, als dasselbe mehr Brom enthält und durch Herabsetzung der Herzthätigkeit leicht tödtlich wirken kann. Einen derartigen Fall von Verabreichung von Bromätbylen anstatt Bromäthyl berichtet $\left.\mathrm{Hirsch} \mathrm{sc}^{1}\right)$. Es trat heftiges Erbrechen mit Collapserscheinungen auf, jedoch war im Uebrigen der Ausgang gïnstig.

Zur Narkose bediene ich mich derselben mit Flanell überzogenen Maske, welche beim Chloroformiren verwendet wird. Die Maske wird reichlich übergossen und dann fest vor Mund und Nase des Patienten gehalten. Das Angstgefühl, welches bei den ersten Chloroforminhalationen fast regelmässig auftritt und der infolge dessen hervorgerufene Widerstand gegen das Auflegen der Maske sind beim Bromäthyl sehr gering und fehlen häufig sogar vollständig. Einige Patienten, welche früher schon einmal chloroformirt waren, gaben an, dass die Bromäthylnarkose weit weniger quälend sei, andere fanden die Inhalationen sogar sehr angenehm. Nach wenigen Athemzügen empfinden die Krankeu ein Schwindelgefühl, welehes bald vorübergeht.

Gewöhnlich ist schon jetzt Analgesie eingetreten, während das Bewusstsein und die Tastempfindung noch vorhanden sind. Die Beribrung mit dem Instrument wird wahrgenommen, aber ohne Schmerz zu verursachen, Fragen werden richtig beantwortet. $\mathrm{Za}$ Anfang habe ich stets in diesem Stadium, welches gewöhnlich schon nach einmaligem reichlichen Durchtränken der Maske erreicht wird, operirt, aber infolge eines unvollkommenen Erfolges giesse ich jetzt mehrere Male auf, bis sich die Maske mit den oben erwähnten Eiskrystallen bedeckt. Das Bewusstsein ist dann in der Regel völlig erloschen. Zuweilen kommt es vor, dass die Patienten während des Eingriffes schreien; nach dem Erwachen sind sie sich dessen aber nicht bewusst und geben an, auch keinen Schmerz gefiuhlt zu haben. Während der Narkose sind die Cornealreflexe erbalten. Es ist dies gewissermaassen ein Uebelstand, da uns das Symptom, welches beim Chloroform die Vollendung der Narkose angiebt, bier fehlt, aber durch probatorische Stiche mit der Stecknadel, dureh Kneifen der Haut, aber nach kurzer Uebung auch ohne jene Merkmale lässt sich bald entscheiden, wann die Betäubung eine genügende ist. Der

1) Das Bromäthyl und die Bromäthylnarkose. Therapeutische Monatshefte. 1888. S. 556. 
Puls ist in einigen Fällen gar nieht, in anderen ein wenig beschleunigt, bleibt aber regelmässig und voll. Die Athmung: nimmt kaum merklich $z u$, die Athemzilge werden zuweilen flacher. Eine Röthung des Gesichts, wic sie Asch'1) erwähnt, babe ich nur in sebr wenigen Fällen constatiren können, dagegen waren die Pupillen cbenfalls, wie bei seinen Beobachtungen, dilatirt.

Das Verhalten der Muskeln war versehieden. In der geringeren Anzahl der Fälle trat Erschlaffung ein, so dass die Kranken vom Stubl zu sinken drohten; in der Mehrzahl blieb der Muskeltonus annähernd normal, einige Malc kam es zu förmlich tetaniseber Versteifung, besonders der Extremitätenmusculatur. Trotzdem habe ich mich niemals genöthigt gesehen, den Patienten auf den Operationstisch zu lagern, sondern habe den Eingriff stets am sitzenden Kranken rollenden können.

$\mathrm{Zu}$ einer Narkose habe ich durchschnittlich $20 \mathrm{Grm}$. Bromäthyl verbraucht und ich befinde mich damit in annähernder Uebereinstimmung mit $\mathrm{Asch}$ (a. a. 0.) und Szumann (a. a. 0.), von denen der eine 5,0-30,0, der andere 10,0-30,0 als Dosis angiebt. Die längste Dauer einer Narkose betrug 20 Minuten. Es handelte sich in diesem Falle um eine Durchtrennung des $\Lambda$ mboss-Steigbügelgelenkes und Extraction des Amboss. Die anderen Narkosen waren weit kurzer. Asch berichtet, dass nach einer Zeit von 15 Minuten die anästhesirende Wirkung nachlasse und auch bei fortgesetztem Aufgiessen nicht wieder eintrete, ebenso dass eine Narkose, wenn sie frühzeitiger unterbrochen ist, durch erneute Anwendung des Bromäthyl nicht wieder erreicht werde. Uebrigens lässt sich, wenn die Bromäthylnarkose unsicber wird, ohne Schaden für den Kranken die Chloroformnarkose anschliessen. Das Erwachen ans der Bromäthylnarkose ist meist ein schnelles. Wenn es nicht spontan nach dem Aufhören der Inhalationen erfolgt, so lässt es sich durch Anrufen oder leichtes Schatteln erreichen. Die Mehrzahl der Kranken ist sofort bei Bewusstsein und erkennt die Situation. Bei einzelnen wirken aber die ldeen und Gedanken, mit welchen sie sich während der Narkose befasst haben, noch einige Secunden nach, um allmählich einem vollständig klaren Bewusstsein Platz zu machen. Beunruhigende Symptome habe ich ebensowenig wie Asch und Szumann während oder nach der Narkose gesehen.

1) Veber Bromäthyl. Therapeut. Monatshefte. 1887. S.54ff. 
Erbrechen während der Narkose ist selten, nach der Narkose häufiger, aber immer noch gering im Vergleich zum Chloroform. Die mit dem Erbrechen verbundene Uebelkeit verliert sich in der Regel nach einigen Stunden und dauert nur in ganz seltenen Fällen bis zum nächsten Tage.

Anfgefallen ist mir unmittelbar nach dem Erwachen, was ich anderweitig nicht erwähnt finde, bei einzelnen Patienten, und zwar bei Kindern, eine Insufficienz der Beinmuskeln, welche das Gehen unmöglich machte, aber nach wenigen Minuten regelmässig verschwand. Die Exspirationsluft der Narkotisirten riecht noch 1 bis 2 Tage melur oder weniger nach Phosphor. Jedoch belästigt diese Erscheinung die Kranken in keiner Weise, und die Umgebung merkt in den meisten Fällen, wenn sie nicht besonders darauf aufmerksam gemacht werden, gar nichts davon. Eine entzündliche Affection der Respirationsorgane nach Anwendung ron Bromäthylinhalationen, wie sie anderweitig beoabachtet ist ${ }^{1}$ ), habe ich niemals constatiren können.

Die Bromäthylnarkose vollzieht sich, wie aus der obigen Beschreibung hervorgeht, in der Regel ohne Excitationsstadium. Eine unangenehme Ansnabme hiervon machen Potatoren. Hier tritt schon bald nach den ersten $A$ themziugen eine erhebliche Erregung ein, so dass ohne Anwendung ron Gewalt ein Fortsetzen der Narkose zur Unmöglichkeit wird. Ich habe denn auch in solchen Fällen auf die Fortsetzung verzichtet, den Kranken etwas zur Ruhe kommen lassen, aber auch dann noch den Eindruck gewonnen, dass die Schmerzempfindung nicht unerheblich herabgesetzt war.

Bei Lungen- und Herzkranken, Nierenleidenden u. s. w. wird mân ebenso wie beim Chloroform nur mit der grössten Vorsicht vorgehen, oder wenn irgend möglich, lieber ganz auf die Narkose verzichten. Erwähnen will ich, dass unter vielen Hunderten von Bromäthyluarkosen in der Literatur 2 Todesfälle bekannt sind, von denen der eine eine Phthisica, der andere eine an keiner nachweislichen Organerkrankung leidende Frau betraf. ${ }^{2}$ )

Nach dem bisher Gesagten besitzen wir in dem Bromäthyl ein Mittel, welches bei kurzdauernden, schmerzhaften Eingriffen, wie sie gerade in der Obrenheilkunde so häufig vorkommen, als

1) Muller, Berl. klin. Wochenschrift. 1883. $\mathrm{Nr}, 44$.

2) Diese Mittheilungen stammen aus dem Jahre 1880, seitdem sind, soweit mir bekannt, keine weiteren Todesfälle zu verzeichnen. 
Anästheticum am Platze ist. Es fübrt eine schnell eintretende Narkose herbei, ist für die Kranken weniger unangenehm als Chloroform, sowohl bei der Application, wie in den Nachwirkungen, bedarf keines so umfangreichen Apparates und kann ohne sachkundigere Assistenz angewendet werden. Die Nachtheile, welche es dem Chloroform gegenuber hat, nämlich das Fehlen des Cornealreflexes und die Unsicherheit der Wirkung bei länger dauernder Narkose, fallen nicht allza sehr ins Gewicht, da bei einiger Uebung der Moment, wo der Eingriff begonnen werden darf, leicht festzustellen ist, and da im letzteren Fall, wenn die Narkose verlängert werden soll, Bromäthyl mit dem Chloroform ohne Nachtheil vertauscht werden kann.

Eines der vielgenanntesten unter den neneren antiseptischen Mitteln ist das Creolin. In den ersten Publicationen als ein Mittel dargestellt, welches vor den bisher bekannten ausser der sicheren keimvernichtenden Eigenschaft den Vorzug der Ungiftigkeit besässe, hat es jetzt nicht an Mittheilungen gefehlt, welche jene Eigenschaft in Frage stellen. ${ }^{1)}$ Das Creolin, der Firma William Peurson \& Co., ein Product der trockenen Destillation der Steinkoble, dessen Constanz aber nicht über jeden Zweifel erbaben zu sein scheint, ist eine schwarzbraune, zähe, syrupähnliche Flissigkeit von sebr durchdringendem, an Steinkohlentheer erinnernden Geruch. In Wasser gebracht bildet es eine milchähnliche Emulsion, welche bei längerem Stehen sich etwas bräunt und einzetne grössere ölige Tropfen abscheidet. Ausser seiner keimvernichtenden Wirkung soll das Mittel ein vorzügliches Desodorationsvermögen besitzen, Granulationsbildung und Vernarbung befördern, secretbeschränkend und blutstillend sein. Ueber Verwendung des Creolins in der Otiatrie liegt, soweit mir bekannt, bisher nur eine Publication von Eitelberg ${ }^{2}$ ) vor. Behandelt wurden hauptsächlich dort acute Eiterungen durch Ausspïlen mit einer Lösung von 10 Tropfen Creolin auf ${ }_{1 / 2}$ Liter Wasser und wurden einer verhältnissmässig schnellen Heilung entgegengefibrt. Ich habe das Creolin fast ausschliesslich bei chronisehen Eiterungen angewendet, da meiner

1) Nendörfer, Das Creolin und seine therapeutische Verwendung. Internat. klin. Rundschau. Wien 1888. Nr. 17 u. 18. Therapeut. Monatshefte. 1888. S. 480 u. 573 .

2) Ueber therapeutische Verwendung des Creolins in der Otiatrie. Wien. med. Presse. 1888. Nr. 13. 
Meinung nach die acuten Formen keinen sehr geeigneten Maassstab für die Brauchbarkeit eines Mittels abgeben. Denn auch bei sorgsamer Reinigung mit den indifferentesten Mitteln, Abhaltung von Schädlichkeiten und zweckmässigem Verhalten heilen acute Eiterungen in kurzer Zeit aus. Wenn die Heilung nun nach Anwendung von diesem oder jenem Medicament erfolgt, so geht darans doch noch nicht hervor, dass sie wegen dessen erfolgt ist.

Anders die chronischen Eiterungen. Sie können ja, besonders wenn sie nicht complicirt sind, aussehliesslich durch subtile Reinigung geheilt werden, erfordern aber dazu fast ausnahmslos lange Zeit. Gelingt es nun, bei Anwendung eines Mittels in kurzer Zeit bei einer grösseren Reihe einschlägiger Fälle Heilung: zu erzielen, so sind wir berechtigt, die Heilung auf Kosten jenes Mittels zu setzen.

Gemäss der Untersuchungen Eis enberg's '), denen zufolge erst von einer 1 proc. Lösung ein einigermaassen sicher keimtödtender Effect zu erzielen ist, versuchte ich eine solche Concentration zu Ausspïlungen anzuwenden. Ich musste aber sogleich davon abstehen, da sie ein unerträgliches Brennen hervorrief. Erst in einem Verhältniss von 2:1000 wurde das Mittel vertragen, obwohl es auch in dieser Stärke noch brennt. Störend war der Umstand, dass wegen der Undurchsichtigkeit der Spiulflussigkeit eine Controle der Beschaffenheit des heransbeförderten Secretes unmöglich wurde. Ich spritzte deshalb zu Anfang mit Kochsalzlösung und benutzte das Creolinwasser zum Nachspülen. Bei den Eiterungen, welche mit Fötor verbunden waren, konnte eine Verminderung desselben constatirt werden. Doch war der Effect kein nachbaltiger und versehwand sofort beim Aussetzen des Mittels. Es zeigt sich hier einmal wieder deutlich, dass Desinfection und Desodoration sich durchaus nicht decken.

In einer Anzabl Erkrankungen war eine secretvermindernde Wirkung des Creolins nicht zu verkennen. Es handelte sich hier in der Regel um Eiterungen ohne Complication, bei denen grosse Perforation, Absonderung eines vorwiegend schleimigen Secretes, Feblen ciner erheblichen Schleimhautschwellung sich fanden. Sehr schön konnte ich einige Male beobachten, wie bei totalem Defect des Trommelfells vom Rande her sich bläulichweisse Streifen Epidermis vorschoben, zu Leisten vereinigten, welche zwischen sich röthliche Inselchen noch nicht epidermisirter

1) Ueber desinficirende Wirkung und die praktische Anwendungsweise des Creoiin. Wiencr med. Wochenschr. 1888. Nr. 17, 18, 19. 
Schleimhaut iubrig liessen, die allmählich sich immer mehr verkleinerten, bis endlich nach einigen Wochen die ganze Fläche epidermisirt, die Secretion aufgehoben war. Ich möchte glauben, dass dieser Epidermisirungsprocess bei Totalverlust des Trommelfells leichter von Statten geht, als bei vorbandenen Resten desselben. Ich habe wenigstens bei Otorrhöen mit grösseren oder kleineren Perforationen, welche die Betrachtung der Pankenschleimhaut ermögliehen, nach Sistiren des Ausflusses die Auskleidung des Cavum tympani gewöhnlich als eine leicht röthliche, mit deutlichen Gefässen versehene Membran gefunden, welcher das trockene weisse, gefässlose Aussehen der Epidermis nicht eigen war.

Ich hatte den guinstigen Einfluss des Creolins auf die Benarbung auch nach anderer Richtung bin zu beobachten Gelegenheit, nämlich bei tiefgreifenden Ulcerationen in der Haut der Nase und der benachbarten Gesichtspartien. Die Kranke, um welche es sich hierbei handelte, batte seit Monaten daran gelitten, neben allerlei äusserlichen Mitteln innerlich Jodkali erlalten, da der Verdacht einer specifischen Erkrankung nicht unbegründet war, ohne dass eine Besserung eingetreten wäre. Ich reinigte die Geschwtire mit einer 1 proc. Creolinlossung, verordnete Auflegen von Compressen mit gleicher Lösung durchtränkt und war aufs Angenehmste tiberrascht, nach Verlanf von 8 Tagen den grössten Theil der Ulcerationen fest vernarbt zu sehen. Die an Ausdehnung bedeutenderen zeigten gute Granulationsentwicklung, am Rande einen deutlichen bläulichen Epithelsaum, von dem aus Fortsätze über die granulirende Fläche ziehen. In kurzer Zeit war auch hier Vernarbung eingetreten.

Aber nicht in allen Fällen nncomplicirter chronischer Eiterung konnte die günstige Wirkung des Creolins constatirt werden; eine Anzahl blieb trotz Wochen lang fortgesetzter Creolinbehandlung unbeeinflusst, leistete aber auch den anderen iblichen Mitteln den hartnäckigsten Widerstand. Jedenfalls glaube ich aber die nach Creolinanwendung eingetretenen Heilungen, welche dann in der Zeit weniger Wochen erfolgten, nicht nur als rein zufällige auffassen zu dürfen, sondern sie dem Mittel zuschreiben zu können. Za Injectionen per tubam habe ich das Medicament wegen seines hässlichen Geschmackes nicht verwendet, sondern dazu die übliche $3 / \wedge$ proc. Kochsalzlösung genommen. Irgend welche ublen Wirkungen, wie Reizungen und Entztīndungen des Gehörgangs, Ekzembildung u.s. w. habe ich nicht zu verzeichnen gehabt. 
Wir sahen, dass die Creolinlösung aus bestimmten Gründen nur in einer Concentration angewendet werden konnte, welche zur schnellen Vernichtung von pathogenen Keimen zu schwach war. Wenn wir nun aber doch Erfolge erzielen, so verdanken wir dies eben anderen, als den antiseptischen Eigenschaften des Mittels. Infolge unserer Kenntniss, dass Krankbeiten wohl ausnahmslos hervorgerufen werden durch die Invasion niederer Organismen, ist das Bestreben der Therapie auf Vernichtung der eingedrungenen Elemente gerichtet und vergisst leicht dariber, dass noch andere Momente vorhanden sind, wo sic ibren Hebel ansetzen kann. Wie eine gesunde Schleimhaut nicht obne Weiteres durch Uebertragung irgend eines pathogenen Keims erkrankt, sondern erst in irgend einer Weise rorbereitet sein muss, damit jener Keim sich darauf zu entwickeln und seine deletäre Wirkung zu entfalten vermag, so ist es denkbar, dass umgekebrt dic Lebensthätigkeit und Entwicklung der Mikroben an Intensität und Extensität abgenommen haben, ohne dass die Scbleimbaut anatomisch und functionell zur Norm zurückkehrt. Durch die Einwanderung sind die Circulations-, Innervations- und Secretionsverhältnisse verändert worden, und dieser veränderte Zustand bleibt stationär.

Ich habe hier jene Fälle chronischer Eiterung im Auge, welche mit grosser Perforation, ohne Knochenaffection, ohne Neigung zu Granulationsbildung, lediglich durch eine erbebliche, vorwiegend schleimige Secretion sich charakterisiren. Auch Stacke ${ }^{1}$ ) glaubt, dass diese Form, welche primär wobl durch Pilze bedingt sei, in dem späteren Stadium durch eine Ernährungsstörung der Schleimhaut unterbalten wird, um so mehr, da im Secret grössere Mengen von Mikroorganismen nicht zu finden wären. Hier ist von starken Antisepticis wenig oder gar niehts zu boffen, sondern von solchen Mitteln, die eine Abnahme der Hyperämie oder eine Beschränkung der Secretion hervorzurufen im Stande sind: adstringirende und alterirende, um mich dieses althergebrachten, wenn auch sehr allgemeinen und nichts erklärenden Ausdruckes zu bedienen.

Das bestätigt anch die Erfabrung. Durch Anwendung starker Sublimatlösungen wird selbst nach längerer Dauer selten oder nie Heilnng erreicht. Dagegen sind namentlich, wenn es sich um erheblichere Schwellungszustände handelt, mit der von

1) Die Behandlung der Otorrhoe mit Borsäurepulver. Deutsche med. Wochenschrift. 1887 . Nr. 49 u. 50. 
Schwartze angegebenen kaustischen Lapislösung glänzende Erfolge zu erzielen.

Umstimmende Wirkung schen wir in den Fällen, wo es sich weniger um Schleimbautschwellung, als um Hypersecretion handelt, eintreten wach Alkohol und Borsäure. Ich möchte Letzteres ganz besonders hervorheben, um zu beweisen, dass es mir durchaus fern liegt, die Borsäure in Pulverform absolut aus dem therapeutischen Heilschatz der Otiatric verbannt zu wünschen. Ich habe mich in fruheren Artikeln nur dabin erklärt, dass es unrichtig sei, Borpulver bei jeder Eiterung ausnahmslos anzuwenden, da es in manchen Fällen schädlich werden kann, z. B. bei enger Perforation durch Hinderung des Secretabflusses und dadurch bedingter Eiterretention, in anderen, z. B. stinkenden Otorrhöen mit Erkrankung des Knochens, wegen seiner sehr geringen zersetzungswidrigen Eigenschaft unwirksam und aussichtslos sich erweist.

Was die antizymotische Kraft der Borsäure anlangt, so hat Prof. Walb ${ }^{1}$ ) dargethan, dass dieselbe im Stande ist, frisch bereitetes Blutfibrin von Fäulniss frei zu halten, und will das Mittel deshalb bei Eiterungen angewandt wissen, um den Eintritt ciner Fäulniss zu verhindern. Dass die Borsäure kolyseptisch ${ }^{2}$ ) wirkt, ist zweifellos und geht auch aus den Arbeiten Koch's zur Genüge hervor. In dieser Absicht bei nicht complicirten Otorrhoen angewandt, mit Ausscheidung aller Fälle, wo Retention zu befürchten ist, ein Ereigniss, welches auch Prof. Walb cintreten sah, hat die Borsäure ihre Berechtigung. Es sind dies dann eben jene Fälle, bei welchen, wie ich oben andeutete, das Mittel oft Heilung erzielt, allerdings, wie ich glaube, weniger durch Verhinderung der Fäulniss, als durch eine gewisse alterirende Wirkung. Wenn aber Fäulniss besteht, wie es Knochendestructionen mit sich bringen, so ist nach vielseitigen anderen und auch meinen Erfahrungen von der antizymotischen Kraft der Borsäure nichts zu erreichen. Deshalb scheint mir der Name antiseptische Behandlung für die Borsäurebehandlung nicht gerechtfertigt, da er bei weniger mit den Verbältuissen Vertrauten den Glauben erwecken muss, dass die Borsäure ein unfehlbares Mittel gegen alle eitrigen Processc, ob mit oder ohne faulige Zersetzung einhergehend, sein muss.

1) Ueber Fistelöffnungen am oberen Pole des 'Trommelfells. Dies. Arch. Bd. XXVI. S. $211 \mathrm{ff}$.

2) Der Ausdruck findet sich gebraucht von Guillery, Beiträge zur Sublimatfrage. Deutsch. med. Wochenschrift. 1858. Nr. 21. 
Wenn ich mir die Wirkung des Creolin zu Stande kommend denke in der Weise, dass es ähnlich wie die balsamischen Mittel die seeretproducirenden Factoren zum Angriffspunkt nimmt, so finde ich diese Ansicht unterstützt durch die Resnltate, welche ich bei der Behandlung von Rhinitiden mit dem Mittel erzielte. Ich benutzte es fast anssehliesslich bei den Formen, bei welchen das Seeret eine vorwiegende Tendenz zar Eintrocknung zeigte, in einer Lösung, welche noch schwächer als beim Obr gewählt werden musste, um das Brennen einigermaassen erträglich zu machen. War die Krustenbildung nicht sehr hochgradig, so konnte schon nach einigen Tagen eine geringere Neigung zum Eintrocknen bei täglicher ein- oder mehrmaliger Reinigung constatirt werden. Das Secret blieb fliussig, von schleimig-eitriger Beschaffenbeit. Nach einigen Wochen nahm auch die Menge desselben ab und die Farbe näherte sich mehr und mebr der Norm. Bei mittelsehweren Fällen kam es so weit, dass nur aller paar Tage eine Ausspülung nöthig wurde; ganz sehwere Fälle wurden allerdings nur wenig beeinflusst. Meine Resultate bei Rhinitiden sind zwar nicht ganz so günstig wie die von Plesk off ${ }^{1}$ ) mitgetheilten, dürften aber doch immerhin zu weiteren Versuchen mit Creolin auffordern.

Von einer weiteren Eigenschaft des Creolin, der blutstillenden, habe ich mich nicht überzeugen können. Ich habe es bei Hammerextractionen benutat, bin aber wieder zu der Alaunlösung zurickgekehrt. Bei Nasenblutungen liess es mich in einem Falle ebenfalls im Stich, während Eiswasser einen sehr prompten Erfolg erzielte. Als Desinfectionsfltissigkeit zum Einlegen der Instrumente babe ich das Creolin sehr schnell anfgegeben. Ein Uebelstand lag darin, dass man wegen der undurchsichtigen Emulsion nicht schnell das Gewünschte zu finden im Stande war und deshalb genöthigt wurde, die ganzen eingelegten Instrumente zu fassen und das erforderliche auszuwählen. Einen zweiten Nachtheil bildete eine gewisse Schllipfrigkeit, welche die Lösung an den Händen und Instrumenten bervorrief und welche eine sichere Führung derselben fast zur Unmöglichkeit machte. Von der granulationsbefördernden Wirkung des Creolins babe ich mich bei der Behandlung von Wunden, die zum Zweck der Eröffinung des Warzenfortsatzes angelegt wurden, mehrfach

1) Therapeutische Versuche mit Creolin. Therapeutische Monatshefte. 1888. S. 460. 
iiberzeugen können. Blossliegender Knochen, welcher bei Sublimatverband keine Tendenz zur Granulationsbildung zeigte, bedeckte sich nach Anwendung von Creolintampons in wenigen Tagen mit reichlichen, gesunden Granulationen; ein thermässiges Wuchern derselben, welches Touchirungen mit Lapis verlangt hätte, trat nur selten ein.

Fassen wir unsere Resultate kurz zusammen, so haben wir in dem Creolin ein Mittel, welches zwar seine unangenehmen Eigenschaften hat: den wenig schönen Geruch, die Undurchsichtigkeit der wässrigen Mischung, das Brennen bei der Application, das aber doch für manche Formen von Eiterungen nicht unwirksam zu sein scheint und deshalb, wenn es auch nicht wesentlich mehr leistet als andere schon bekannte Mittel, nicht kurzer Hand abgewiesen zu werden verdient.

Besonders mag es sich empfehlen, dasselbe da zu verwenden, wo dem Patienten die Ausspülungen grösstentheils äberlassen werden mïssen, weil seine relative Ungiftigkeit grösseres Unglïck nicht hervorrufen kann.

In der Aetiologie zahlreicher Ohrkrankheiten spielen die Erkrankungen der Nase und des Rachens bekanntlich eine grosse Rolle und besonders sind es die katarrhalischen Entztindungen der Schleimbäute der oberen Respirations- und Deglutitionsorgane in allen ihren Stadien, welche consecutive Katarrhe der Tuba und der Paukenhöhle hervorrufen. Es ist deshalb eine Beseitigung jener eine Nothwendigkeit, wenn eine dauernde Heilung des Ohrleidens erreicht werden soll. In der Regel sind aber die Erkrankungen der Nasen- und Rachenschleimhaut, wenigstens in der chronischen Form, sebr hartnäckig und recidiviren nach erfolgter Heilung doch gern.

Schon seit Langem waren mir die häufigen Klagen von Kranken, welche an jenen Zuständen litten, über kalte $\mathrm{F} u \mathfrak{u s e}$ aufgefallen und hatten mich zu der Ueberzengung gebracht, dass wohl ein Causalnexus zwischen diesem Uebel and jenen Affectionen bestehen miisse. Ich batte deshalb die Neigung zu kalten Füssen dureh reizende Fussbäder event. bekämpfen zo mïssen geglaubt, allerdings obne nennenswerthen Erfolg. Interessant war mir daher eine Arbeit von Branda ${ }^{1}$ ), in weleher der Verfasser

1) Ueber die habituelle Hyperhidrosis pedum. Sonderabdruck der Deutschen Medicinalzeitung. 1886. Nr. 68 u. 69. 
die Neigung zu kalten Füssen als Folge von Hyperhidrosis pedum hinstellt und ein Mittel dagegen empfiehlt, welchem er den Namen Liquor antihidrorrhoicus beigelegt hat. Es gehört in die Reihe der gechlorten Aetherarten und besitzt die Eigenthümlichkeit, dass es für die Haut durchgängig ist. Die bänfige Coincidenz von Katarhen der Nase und des Rachens und Hyperhidrosis betont der Verfasser ganz besonders. Ich habe seitdem bei den einschlägigen Erkrankungsformen der Halsorgane und Nase stets auf das Vorhandensein von kaltem Fuss oder Schweissfuss examinirt, wohl ausnahmslos bejahende Antworten erhalten und deshalb Versuche mit dem Brandau'scben Liquor gemacht. Die Anwendungsweise des Mittels ist eine sehr einfache. Nachdem die Fisse griudlich mit warmem Wasser bearbeitet und vollständig abgetrocknet sind, giesst man in eine grosse flache Schüssel (kein Metall, welches angegriffen wird) so viel von dem Liquor, dass die Flüssigkeit einige Millimeter hoch steht und setut die Fisse hinein, so dass anfangs nur Ferse und Soble benetzt werden, die Zehen aber ausserhalb bleiben. Nach nngefähr 5 Minuten werden auch diese in die Flïssigkeit gesenkt und eine gleiche Zeit darin belassen. Man hat darauf zu achten, dass der Liquor wohl in den Zwischenräumen der Zehen steht, nirgends aber, weder hier noch an den Seiten des Fusses, den empfindlicheren oberen Theil der Haut bespült. Die Füsse werden, wenn nicht ein Prickeln oder Brennen eine Abkürzung der Einwirkungsdauer erfordert, nach 10 Minnten dauernder Application herausgenommen, nochmals in warmem Wasser abgespílt und aufs Sorgfaltigste getrocknet, damit nicht rückbleibende Theile des Liquor die Haut arrodiren, sodann $1 / 2-1$ Stunde horizontal gelagert. Die gebrauchte Flissigkeit kann wieder benutzt werden.

Da das Mittel grossentheils aus Aether besteht, so ist vor der Annäherung einer offenen Flamme zu warnen. Der nicht unangenehme ätherische Geruch, welcher sich im Zimmer nach Gebrauch des Liquor findet, ist durch Lüftung bald zu beseitigen. Das Verfahren ist anfangs jeden dritten oder vierten Tag zu wiederholen, später in immer längeren $Z$ wischenräumen, bis jede Spur von Hyperhidrosis verschwunden ist. In 8 bis 14 Tagen nach der ersten Anwendung sehält sich die Epidermis in grossen Stitcken ab. Etwaige wunde Stellen sind nicht mit der Flitssigkeit in Berihrung zu bringen, weil sonst ein starkes Brennen hervorgerufen und eine Aetzung der Läsion erfolgt. Schon nach 
den ersten Bädern wird der Fuss trocken, das Kältegefühl ist verschwunden. Bleibt etwa der Erfolg ans, so liegt es fast immer an der ungenanen Ausfubrung der gegebenen Vorschriften.

Seit 1 $1 / 2$ Jabren babe ich in zablreichen Fällen den Liquor antihidrorrhoicus anwenden lassen und constatiren können, dass eine günstige Wirkung auf die Katarrhe der Nase und des Rachens ausgeibt wird, dass besonders hypertrophische Formen von Rhinitis und Pharyngitis nach Wochen, zuweilen auch Monaten, sich zuriackbilden ohne jede Localbehandlung. Die Enscheinungen von Seiten des Ohres, soweit sie abhängig von jenen Affectionen und rïckbildungsfähig waren, verschwanden ebenfalls. Natürlich wird man, um schneller zum Ziele zu kommen, für gewöhnlich die Localbehandlung gleichzeitig neben der Behandlung der Füsse einhergehen lassen. Einige Male verschwand Ohrensausen, welches erst kurze Zeit bestanden, ohne dass ein crheblicherer objectiver Befund zn constatiren war, nach Anwendung des Liquor und trat, wie ich mich nach Verlanf eines halben Jahres tuberzengen konnte, nicht wieder ein. Beiläufig will ich erwähnen, dass anch für die Behandlung der Laryngitiden die Anwendung des Liquor von Bedeutung ist.

Selbstverständlich fehlt es nicht an Fällen, wo der Erfolg nicht dem Gewinsehten entspricht, aber trotzdem halte ich in den Fällen von Ohrencrkrankungen, in welchen gleichzeitig Hyperhidrosis pedum bestebt, einen Versuch mit dem Liquor durchaus für angezeigt.

Selbst wenn es nicht gelingt, den Kranken gerade von dem Leiden $\mathrm{zu}$ heilen, dessentwegen er eigentlich Huilfe sucht, so wird er doch für die gelegentliche Beseitigung eines ihm gleichfalls höehst lästigen Uebels immhin dankbar sein. Nachtheile sind in keinem Falle za erwarten. 